\title{
Investigation of HID-lamp light emission differences for different power supply methods
}

\author{
K. TOMCZUK ${ }^{*}$, P. MAZUREK ${ }^{1}$, L. HEMKA ${ }^{1}$, A. KASPROWICZ ${ }^{2}$, and T. BŁAŻEJCZYK ${ }^{1}$ \\ ${ }^{1}$ Electrotechnical Institute, 28 Mieczysława Pożaryskiego St., 04-703 Warsaw, Poland \\ ${ }^{2}$ Department of Electrical Engineering, Gdynia Maritime University, 81-87 Morska St., 81-225 Gdynia, Poland
}

\begin{abstract}
This paper presents investigation of methods for reducing light pulsation and plasma temperature inside a high intensity discharge (HID) lamp arc tube. Differences found in light emission of an arc tube plasma channel under different power supply methods are presented and discussed in this work. The novelty of the paper lies in systematical investigation of different power converter supply methods and demonstrating that it has a significant influence on plasma temperature in an arc tube. The tested lamp was powered by electronic ballasts controlled by different algorithms, which forced their current waveform. To compare the results, the authors performed measurements on a discharge lamp powered by a standard electromagnetic ballast. The investigation of plasma parameters is based on the optical spectroscopy method. It was shown that by using the appropriate current shape of a high switching frequency supply converter, the plasma temperature of an HID lamp can be reduced almost by half.
\end{abstract}

Key words: plasma temperature, electronic ballast, electromagnetic ballast, lamp current, light flux, light pulsation, high intensity discharge lamp.

\section{Introduction}

The light is one of the main factors affecting the human body. Its quality is very important in various areas of life, including physiology, medicine, stimulation, relaxation, and sense of security. One of the parameters which determines the quality of light flux is its pulsation, and it depends on the method used to supply power to a given light source. Investigations of light flickering generated by light sources are presented in [5] and [6]. Another parameter which determines light emission is the plasma temperature. This parameter has an influence on the spectrum of the emitted light and on its intensity. Decreasing the plasma temperature extends the life of the lamp. Publications [10-20] present theoretical descriptions of a plasma channel and research on this specific state of matter. [2] presents some research on minimization of light flicker, which is dependent on RMS voltage fluctuations in the electrical grid. Light flicker has been also studied in [7], during changes in the shape of a plasma channel, caused by acoustic resonance in the tubes of discharge lamps. In [4], the influence of flicker, at a frequency ranging from few to several dozen $\mathrm{Hz}$, on the brain's electrical activity has been examined using an electroencephalography apparatus (EEG). Light flickering at a frequency of 8-10 Hz results in synchronization of alpha1 and alpha2 brain waves. The same frequency range increases the blood flow to the human eye, which is discussed in [3]. The study of the human eye's response to light flicker, based on a highly developed simulation model is discussed in [1]. None of the referred above publications provides a comparison of the influence of power supply methods on plasma temperature and light flux quality.

*e-mail: k.tomczuk@iel.waw.pl
Therefore, authors decided to perform a research determining the aforementioned parameters of an HID lamp.

\section{Plasma theory}

Plasma is a source of optical radiation. One of the methods used to examine this state of matter is optical spectroscopy. The spectrum of plasma radiation carries a lot of information about atoms or ions, such as the type of gases used in discharge lamps, and the conditions in which they are located. All the processes taking place in radial plasma can be interpreted as a transition between two different quantum energy states. Processes involving the radiation of electrons and atoms (or ions) occur when an electron moves in an electrical field from one charged particle to another, usually an ion. As a result of its interaction with this particle, part of the path of the electron is curved. The temperature inside the arc tube of a discharge lamp is the parameter that determines the appropriate spectral distribution of the energy emitted by the plasma discharge, and the corresponding luminous efficiency. In the radiation spectrum of an HID lamp, there are a number of lines, characteristic of excited atoms. The shape of the spectral line reveals the conditions (pressure and temperature) prevailing in the discharge lamp. Fig. 1 shows a view of luminous plasma in a cold arc tube, whereas Fig. 2 shows luminous plasma in a hot arc tube.

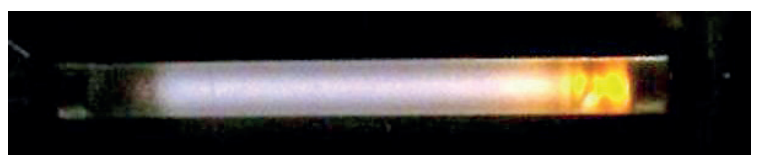

Fig. 1. View of plasma in a cold arc tube 


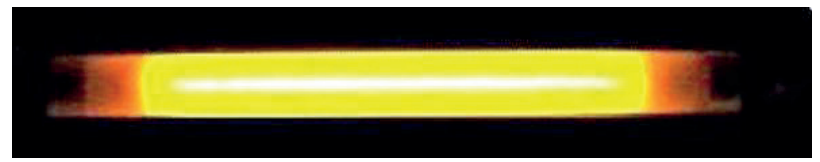

Fig. 2. View of plasma in a hot arc tube

From a theoretical point of view, the radial temperature distribution inside the arc tube of a discharge lamp, can be determined by examining variations, along the radius of the arc tube, in the intensity of the following:

- Optically narrow spectral lines

- Reverse non-resonance lines

- Inverted resonance lines

For each type of line, there is a different theoretical approach for determining the temperature relation $\mathrm{T}(\mathrm{r})$, and in each of these three cases, the following assumptions should be taken:

- The plasma has a cylindrical symmetry

- In the plasma, there is a local thermodynamic equilibrium

- The extension of resonant and non-resonant lines is a result of a collision

Assuming that the above conditions apply, the radiation intensity $I_{v}$ or $I_{\lambda}$ of an arc tube with a small area is determined by the radiation transport equation:

$$
\frac{d I_{v}(x)}{d x}=\mathrm{E}_{v}(\mathrm{x})-\mathrm{k}^{\prime}(\mathrm{x}) \mathrm{I}_{v}(\mathrm{x}) .
$$

Assuming boundary conditions such that $I(R)=0$, this leads to:

$$
I_{v}(x)=\int_{-R}^{+R} \mathrm{E}_{v}(x) \cdot e^{-\int_{x}^{R} k^{\prime}(x) d x^{\prime}} d x
$$

where: $\mathrm{R}$ is the radius of the arc tube, and $\mathrm{E}_{\mathrm{v}}(\mathrm{x})$ and $\mathrm{k}^{\prime}(\mathrm{x})$ are, respectively, the emission and absorption coefficients for $\mathrm{x}$ and $v / \lambda$.

\section{Abel inversion - a survey of an optically thin line}

One method to determine the radial distribution of the temperature and density of particles in a discharge lamp is the study of an optically thin line by optical spectroscopy. A spectral line is considered to be optically thin if:

- The absolute intensity of the line is small, compared to the intensity of black body radiation for a given wavelength $\lambda$ and temperature T;

- For a multiplet:

- The measured ratio of the intensity of the line is close to the predicted theoretical relations of narrow lines. When this ratio begins to approach the values of a black body, the lines are optically wide

- The half-width of a weak line is approximately equal to the width of a strong line
This test method relates to plasma in thermal equilibrium, or at least capable of a local thermodynamic equilibrium. This condition occurs in an arc tube of a high-pressure sodium and metal-halide plasma discharge lamp. It is also assumed that the light source in question has a cylindrical symmetry, and that the extension of the spectral lines is mainly a result of collisions. To achieve the research objective, it was decided to perform an experiment involving the spectroscopic examination of an optically thin line of wavelength $\lambda_{1}=616.1 \mathrm{~nm}$ and $\lambda_{2}=515.4$ $\mathrm{nm}$, in the emission spectrum of a $70 \mathrm{~W}$ high-pressure sodium lamp. The measurements of the mentioned wavelength values were performed by a spectroradiometer Hilger-Engis Monospek 600.

If the test concerns the intensity along the $\mathrm{x}$-direction (Fig. 3), the observed size is derived from the sum of the intensities of all the layers along the y-direction. Consequently, it can be written as:

$$
\begin{aligned}
I_{v}(y) & =\int_{-\sqrt{R^{2}-y^{2}}}^{\sqrt{R^{2}-y^{2}}} \mathrm{E}_{v}(r) d r=2 \int_{0}^{\sqrt{R^{2}-y^{2}}} \mathrm{E}_{v}(r) d r= \\
& =2 \int_{y}^{\sqrt{R^{2}}} \frac{\mathrm{E}_{v}(r) d r}{\sqrt{r^{2}-y^{2}}}
\end{aligned}
$$

where:

$$
\mathrm{E}_{v}(r)=\frac{h c}{4 \pi \lambda} \frac{A_{i k} g_{k}}{Z} \frac{p}{k T(r)} \exp \left(-\frac{E_{k}}{k T(r)}\right) .
$$

Where:

H $\quad-$ Planck's constant

c $\quad-$ speed of light

$\mathrm{A}_{\mathrm{Ik}} \quad-$ probability of transition

$\mathrm{g}_{\mathrm{k}}=2 \mathrm{~J} \_\mathrm{k}+1$ - weight of the statistical energy level in question

$\mathrm{E}_{\mathrm{k}} \quad-$ the energy issue energy

$\mathrm{p} \quad-$ partial pressure of the p-type particles

$\mathrm{Z} \quad-$ split functionality in the first approximation, equal to the weight of the statistical base line test atom

$\mathrm{T}(\mathrm{r}) \quad-$ temperatures proper for the $\mathrm{r}$ ( $\mathrm{r}$ varies from 0 to $\mathrm{R}$, where $\mathrm{R}$ is the radius of the arc tube)

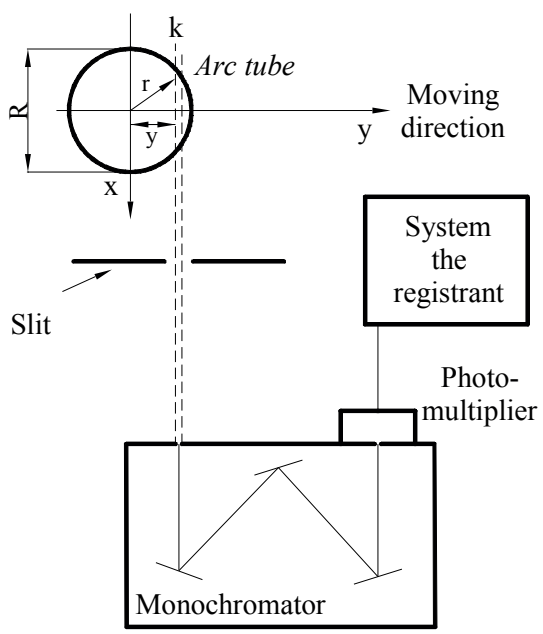

Fig. 3. Schematic system of the arc tube light intensity measurements 
The issue under consideration is essentially the determination of $\mathrm{T}(\mathrm{r})$ based on the measurement of $\mathrm{I}(\mathrm{y})$. The integral type (3) can be reversed using the so-called Abel inversion:

$$
\mathrm{E}_{v}(r)=-\frac{1}{\pi} \int_{r}^{R} \frac{d I(y)}{d y} \frac{d y}{\sqrt{y^{2}-r^{2}}} .
$$

The above integral can be solved by various numerical methods. Here is one of them:

1. The whole plasma region is divided into $\mathrm{N}$ cylinders.

Each $E_{k}(r)$ should be expressed as a sum contributing to $I_{k}\left(y_{k}\right)$ :

$$
\mathrm{E}_{k}\left(r_{i}\right)=\sum_{i=k}^{N-1} b_{i k} I_{k}\left(y_{k}\right) .
$$

This method is preferred by many authors because it gives a relatively good approximation. This is a method that is recursive. The calculations start from the outer layer $\mathrm{f}$ of the arc tube, and proceed towards the center. In order to calculate the coefficients $b_{i k}$, the test area is divided into $\mathrm{N}$ equal cylinders. Then, the desired $\mathrm{E}_{v}$ function (r) can be represented as:

$$
\mathrm{E}_{i}\left(r_{i}\right)=-\frac{1}{\pi} \sum_{k=i}^{N-1} \int_{r_{k}}^{r_{k+1}} \frac{d I_{k}}{d y} \frac{d y}{\sqrt{y^{2}-r^{2}}} .
$$

Assuming that the function $I_{k}$ is an alinear function of the variable $\mathrm{y}^{2}$, the following may be written as:

$$
I_{k} \underset{\substack{w \\ \text { ef }}}{\equiv} I_{k}(y)=I_{k}+\frac{I_{k+1}-I_{k}}{y_{k+1}^{2}-y_{k}^{2}}\left(y^{2}-y_{k}^{2}\right)
$$

Since $\mathrm{y}_{\mathrm{k}}=\mathrm{r}_{\mathrm{k}}$ is the expression, (7) can be written as:

$$
\begin{aligned}
\mathrm{E}_{i} & =\frac{2}{\pi}\left(I_{i} \frac{\sqrt{r_{i+1}^{2}-r_{i}^{2}}}{r_{i+1}^{2}-r_{i}^{2}}+\sum_{k=i+1}^{N-1} I_{k} \frac{\sqrt{r_{k+1}^{2}-r_{k}^{2}}-\sqrt{a^{2}+b^{2}}}{r_{k+1}^{2}-r_{i}^{2}}-\right. \\
& \left.-\sum_{k=i+1}^{N} I_{K} \frac{\sqrt{r_{k}^{2}-r_{i}^{2}}-\sqrt{r_{k-1}^{2}-r_{i}^{2}}}{r_{k}^{2}-r_{k-1}^{2}}\right) .
\end{aligned}
$$

In line with the plasma being divided into $\mathrm{N}$ equal cylinders of width $\omega=\mathrm{R} / \mathrm{N}$ with the result that $\mathrm{r}=\omega \mathrm{I}$ and $\mathrm{I}_{\mathrm{N}}=0$, (9) can be represented in the following form:

$$
\mathrm{E}_{i}=\frac{1}{\varpi} \sum_{k=i}^{N-1} I_{k} b_{i k},
$$

where:

$$
\begin{gathered}
b_{i k}=\left\{\begin{array}{c}
d_{i k} \text { for } k=i \\
d_{i k}-d_{i, k-1} \text { for } k>i
\end{array}\right. \\
d_{i k}=\frac{2}{\pi} \frac{\sqrt{(k+1)^{2}-i^{2}}-\sqrt{k^{2}-i^{2}}}{2 k+i} .
\end{gathered}
$$

Thus, $\mathrm{E}_{\mathrm{i}}(\mathrm{r})$ can be determined using the measured intensity values of $I_{k}$. The above issue can be solved by numerical methods. A special algorithm must be created to determine the leading radial temperature distribution and the density of the plasma particles, studied for two optically thin lines of the same multiplet. Then, $\mathrm{E}_{1}$ and $\mathrm{E}_{2}$ have to be determined for each line separately, and then the ratio can be calculated with the following equation:

$$
\frac{\mathrm{E}_{1 i}}{\mathrm{E}_{2 i}}=\frac{A g_{1}}{A_{g_{2}}} \frac{\lambda_{2}}{\lambda_{1}} \exp \left(-\frac{E_{k 1}-E_{k 2}}{k T\left(r_{i}\right)}\right) .
$$

After doing a conversion of (13), the temperature $\mathrm{T}(\mathrm{r})$ can be calculated.

\section{Power supply methods and the results of high-speed light intensity measurements}

4.1. Description of arc tube power supply methods. Fig. 4. shows current waveforms used in research.

The waveforms are of different frequencies, therefore all of them are expressed on a scale of electrical degrees. A high intensity discharge (HID) lamp was powered by four different current waveforms, generated by four different ballasts. Two of the ballasts (powered in accordance with Method 1 and Method 3) were designed and manufactured by the Electrotechnical Institute (Warsaw, Poland). The topology of converters is halfbridge and full-bridge. Power diodes used in the designed con-

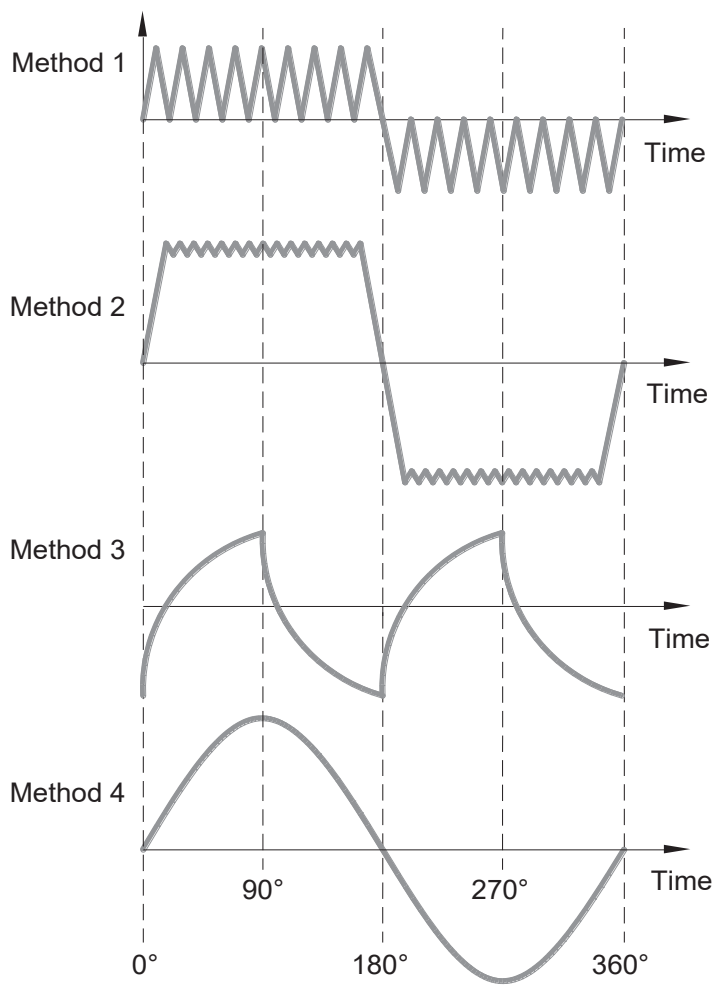

Fig. 4. Description of arc tube current waveforms versus electrical degree scale 
verters are $\mathrm{SiC}$ diodes. Their characteristics are described in [8]. The power loss reduction methods, partially used in control algorithms, are presented in [9]. The other two converters (powered in accordance with Method 2 and Method 4) are used commercially in large-area lighting applications.

The research was performed on a $70 \mathrm{~W}$ ZS-HPS070-27-type sodium lamp, manufactured by Brilum. Power consumption in each of the power supply methods was $\mathrm{P}=70 \mathrm{~W}(\mathrm{U}=70 \mathrm{~V}$, $\mathrm{I}=1 \mathrm{~A})$.

4.2 Description of the high-speed light intensity measurements. High-speed measurements were performed using two methods. The measurements utilizing the first method were taken using a FASTCAM SA X2 Photron high-speed camera, shown in Fig. 5.

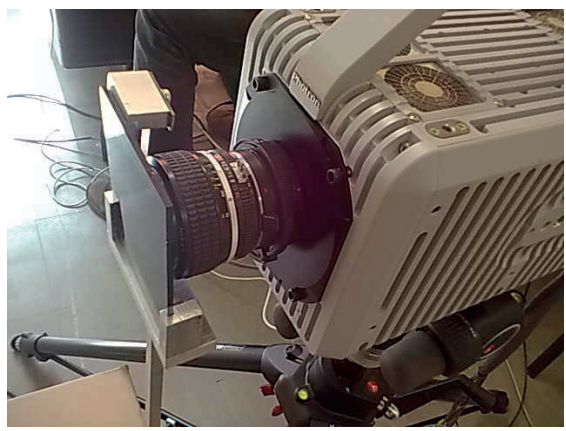

Fig. 5. View of the high-speed camera and the light-blocking filter used during the tests

The pixels in the camera matrix measured the light values in an 8-bit range, where 0 is completely dark and 255 is maximum intensity. The measurements using the second method were taken using a photoelectric transducer with a photodiode, as shown in Fig. 6.

This article presents a comparison of the differences in light emissions for the mentioned power supply methods. For all of the results, the light intensity has been presented in percentage

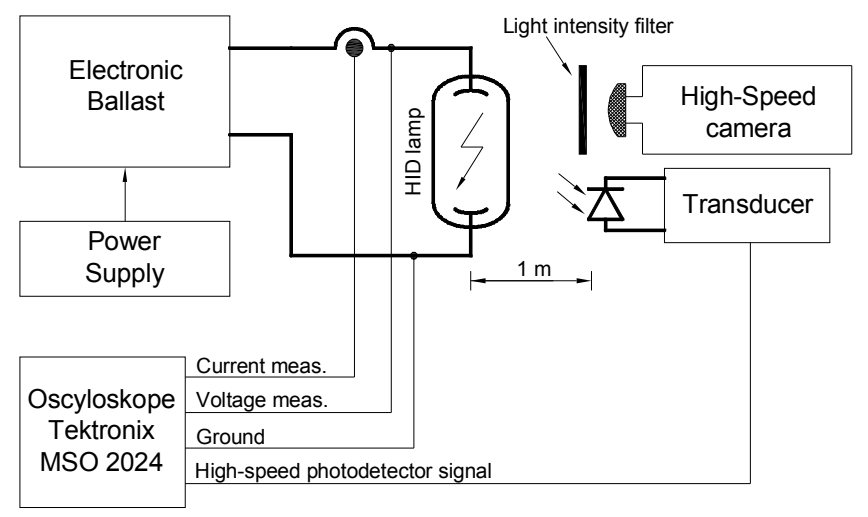

Fig. 6. Test stand for measurements of light flux waveform using a photodiode and a high-speed camera form, where $100 \%$ is the maximum intensity, measured in all four power supply methods. Fig. 6 shows the full diagram of a high-speed light intensity measurement test stand, equipped with a high-speed camera, a high speed photoelectric transducer, an MSO2024 Tektronix oscilloscope, a TCP0030 current probe, a discharge lamp ballast, and a voltage power supply system.

4.3 Measurements of the arc tube light intensity. The highspeed camera shown in Fig. 5 recorded four movies - one for each power supply method of the plasma behaviour in the arc tube, at a speed of 300000 frames per second. This means that a frame was captured once every $3,33 \mu$ s.

Only 5-6 frames from each power supply method were chosen from the set of recorded frames (graphic files) for the light intensity analysis. The frames were taken from a range of current time values from 0 to 180 degrees (Fig. 4). Then, measurements of the pixel values in the 8-bit range (0-255) were performed along the red measurement line shown in Fig. 7(A). The results of the pixel values of the arc tube intensity along the line are shown in Fig. 7(B).

(A)

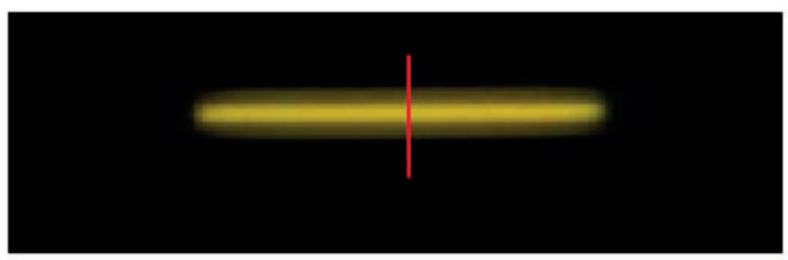

(B)

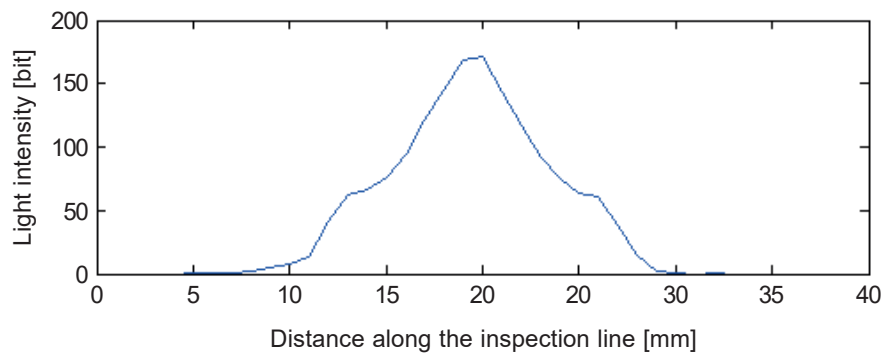

Fig. 7. (A) View of the arc tube with (B) measurements along the red line and the intensity results along the line

4.4. Research results for Method 1. This method consists of powering the discharge lamp with a pulse current with amplitude stabilization and periodic changes of direction. The ballast circuit consists of an EMI filter, an active PFC system, and an inverter designed in half-bridge topology. The circuit allows for power to be supplied to the discharge lamp through the inductor L1. A view of the ballast topology, used in accordance with Method 1, is shown in Fig. 8, where: LP - discharge lamp, and X, Y - ignition coil terminals. After being switched on, the lamp current reaches the predetermined level, and then it is switched off. Before switching the lamp current on for the next pulse, the value of the current is zero. The 


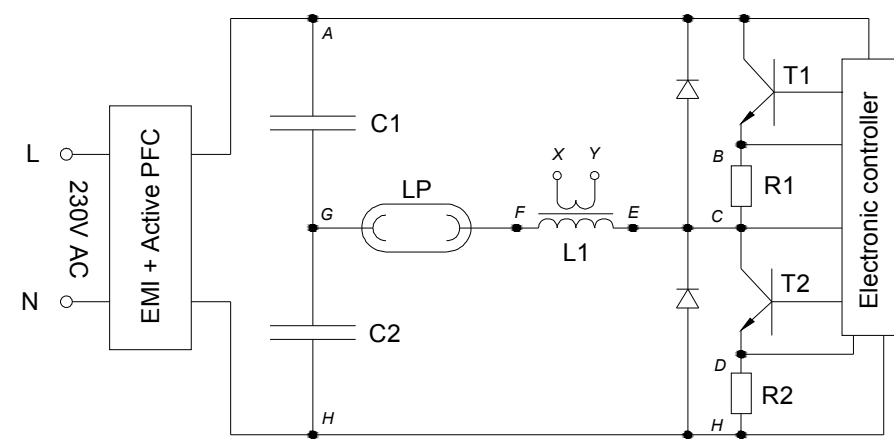

Fig. 8. Electronic ballast (Method 1)

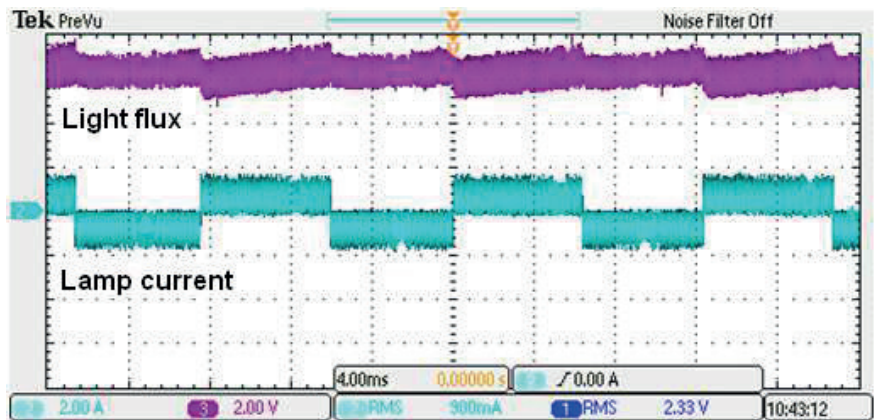

Fig. 9. CH2-lamp current, CH3-light flux (Method 1)

frequency of changes in the direction of the lamp current is approximately $80 \mathrm{~Hz}$, and the current amplitude stabilization frequency is approximately $40 \mathrm{kHz}$. Fig. 10 shows arc intensity across the arc tube and its change over time.

The described power supply method is characterized by a linear distribution of arc intensities between the minimum and maximum current levels. During the current zero-crossing, the arc tube still emits light radiation, which means that the energy is contained in the plasma channel, even if the current is zero.

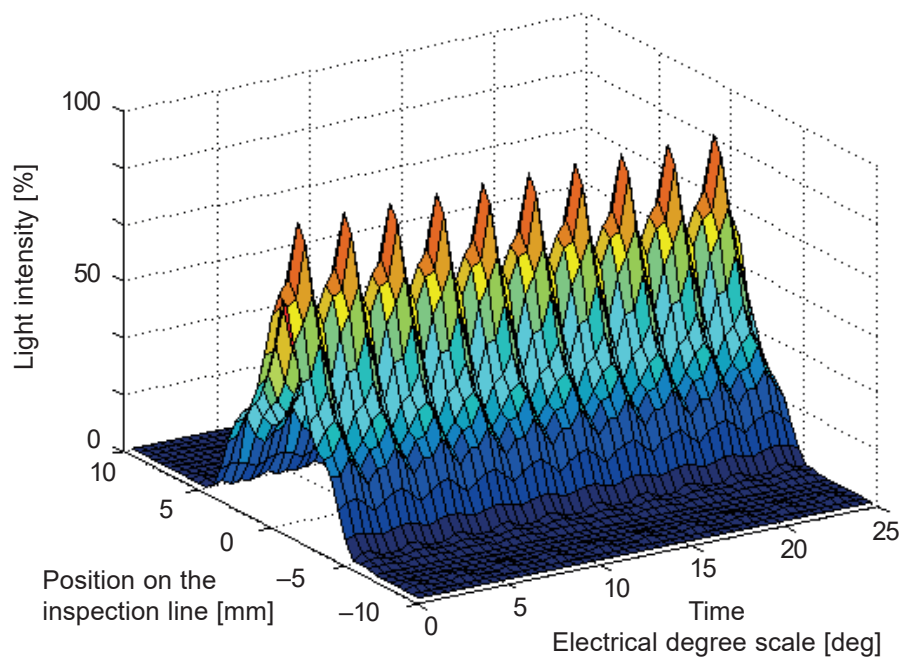

Fig. 10. Arc intensity values across the arc tube for Method 1
4.5. Research results for Method 2. This method consists of powering the discharge lamp with an amplitude-stabilized alternating current. After being switched on, the lamp current is stabilized at a predetermined level for a certain period of time, and after that period of time, the direction of the current changes.

A view of the ballast topology used to supply power to the lamp in accordance with Method 2, is shown in Fig. 11, where: LP - discharge lamp; X, Y - ignition coil terminals; CS - lamp current transducer.

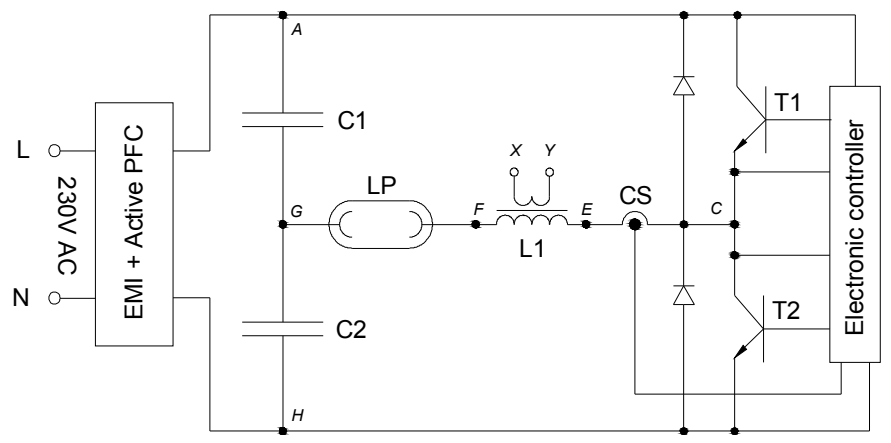

Fig. 11. Electronic ballast (Method 2)

Figure 12 shows the waveforms of the lamp current and light flux emitted by the lamp. The current value is stabilized at a predetermined level, with periodical changes in direction.

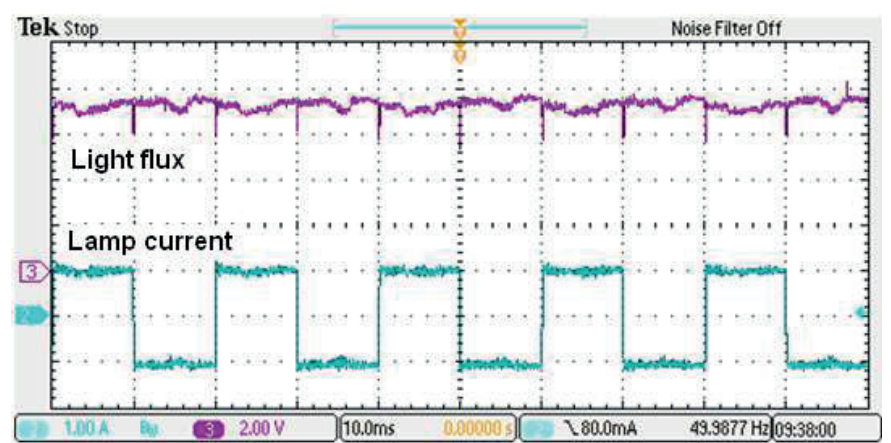

Fig. 12. CH2-lamp current, CH3-light flux (Method 2)

It causes the arc intensity (Fig. 13) to have the same values all the time, except for at the current zero-crossing moment.

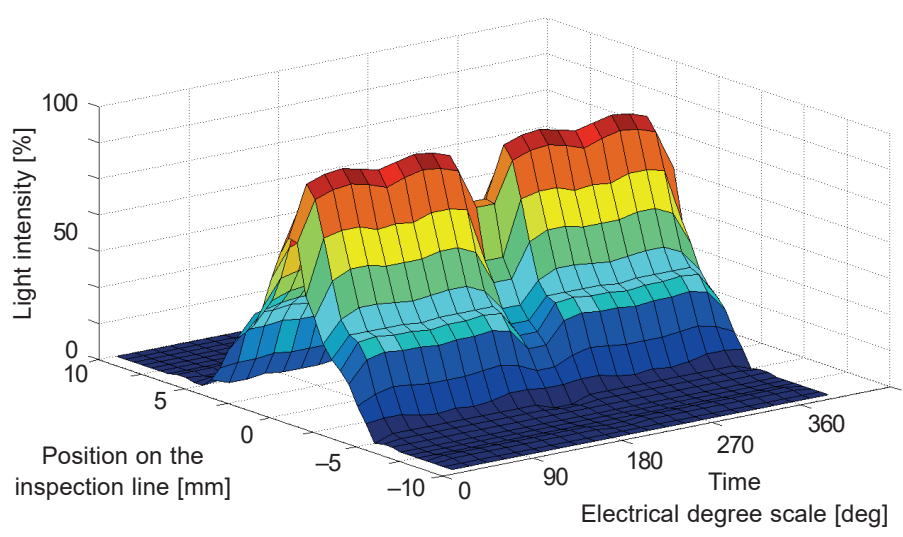

Fig. 13. Arc intensity values across the arc tube for Method 2 
When the current changes direction, the intensity of the light generated by the lamp is reduced by $23 \%$. Under all other conditions, the plasma intensity is constant at a level of $65 \%$ of the maximum measured intensity.

4.6. Research results for Method 3. This method consists of powering the discharge lamp with a high-frequency alternating current with a $50 \%$ duty cycle. Fig. 14 shows a view of the electronic ballast used to supply power to the discharge lamp in accordance with Method 3, where: LP - discharge lamp; X, $\mathrm{Y}$ - ignition coil terminals, CS - lamp current transducer.

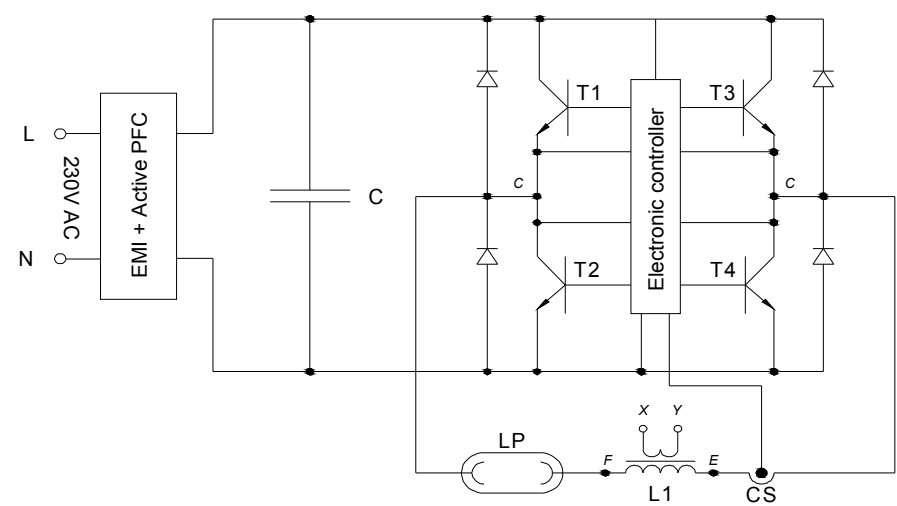

Fig. 14 Electronic ballast (Method 3)

The ballast circuit (Fig. 14) consists of an EMI filter, an active PFC system, and an inverter designed in full-bridge topology, powering the discharge lamp through the inductor L1. Fig. 15 shows the waveforms of the lamp current and the generated light flux. The frequency of changes in the direction of the lamp current is $100 \mathrm{kHz}$. The goal thereof is to make the light emission constant.

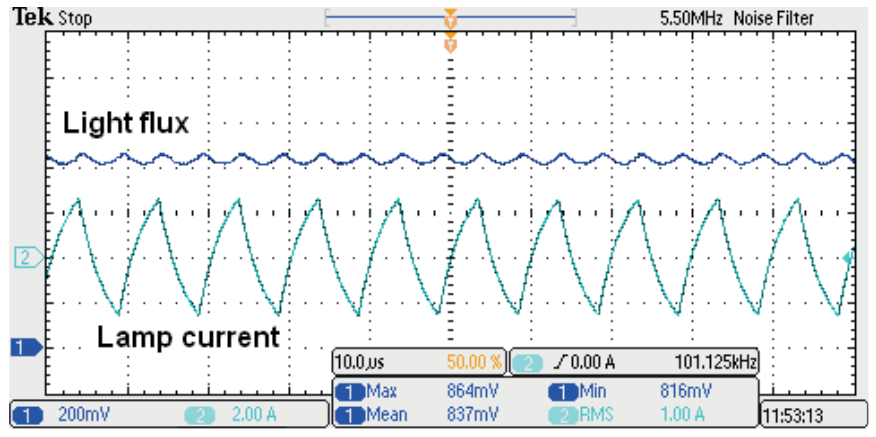

Fig. 15. CH1-light flux, CH2- lamp current (Method 3)

The described power supply method is characterized by the most stable light radiation. The intensity of radiation is $65 \%$ and has this same value for the current peak and zero-crossing.

Fig. 16 shows arc intensity across the arc tube and its change over time.

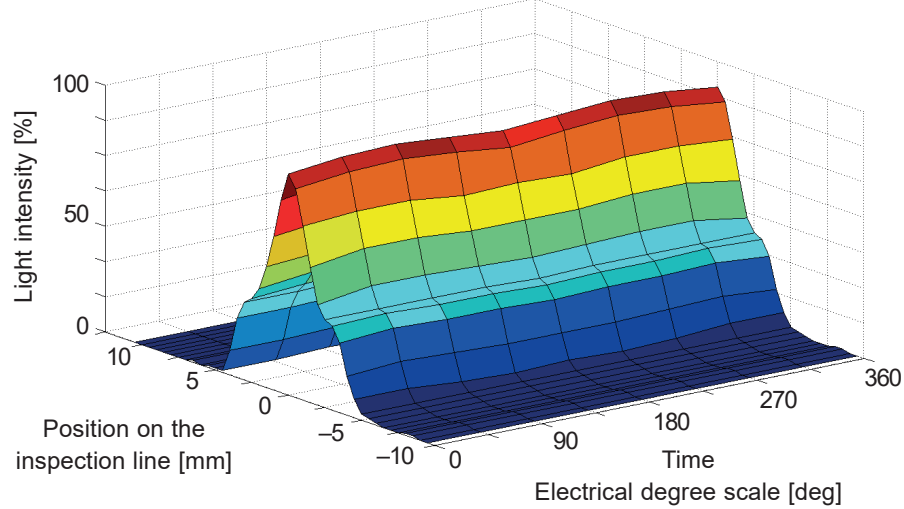

Fig. 16. Arc intensity values across the arc tube for Method 3

4.7. Research results for Method 4. This method consists of powering a high-pressure discharge lamp with a standard electromagnetic ballast with the voltage value of $230 \mathrm{~V}$. The frequency at which the lamp current direction changes is $50 \mathrm{~Hz}$. This method is used as a benchmark comparison of all the methods described above. A wiring diagram of the electromagnetic ballast used to supply power to the lamp in accordance with Method 4, is presented in Fig. 17, where: St - electromagnetic ballast; UZ - ignition circuit.

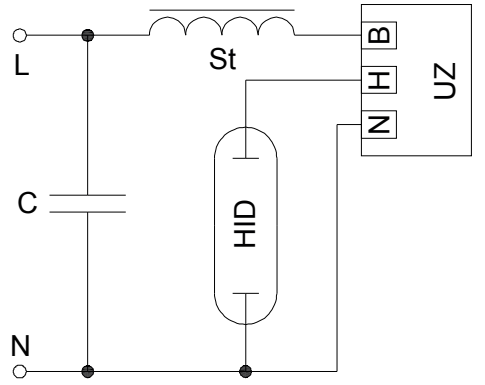

Fig. 17. Electromagnetic ballast (Method 4)

Figure 18 shows the light and current waveforms. At the present moment, it is the most popular method of power supply for discharge lamps. This method of light flux emission is widely used in street and large-area lighting applications. The

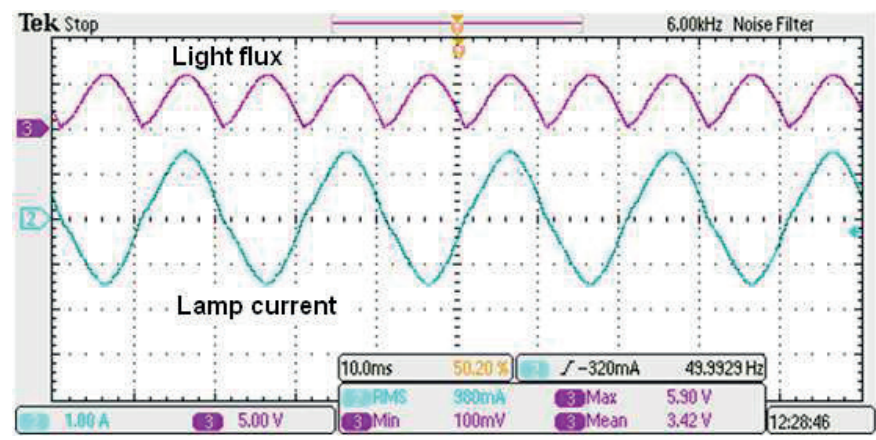

Fig. 18. CH2-lamp current, CH3-light flux. Method 4 
described power supply method causes the biggest differences in light flux emissions.

Fig. 19 shows the arc intensity with the described method. The maximum value is $100 \%$, and it represents the highest radiation of all the power supply methods tested.

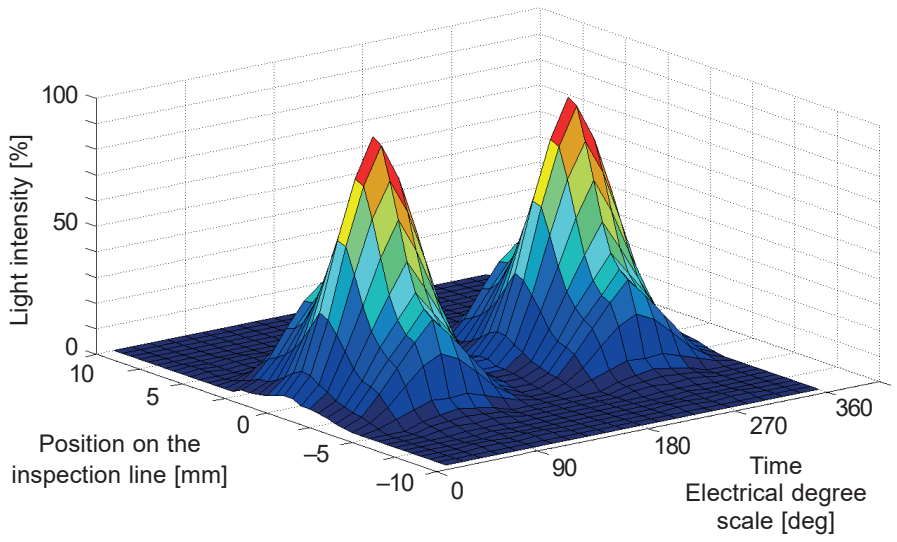

Fig. 19. Arc intensity values across the arc tube for Method 4

The minimum value is $5 \%$, displaying the lowest intensity of all four methods.

\section{Calculation of light flux pulsation}

Although traditionally, two equivalent methods for the calculation of the pulsation value have been used, they give different results. It is absolutely necessary to determine which method is used to calculate a given parameter. In this article, the authors calculate pulsation values using both methods. Table 1 shows the light flux pulsation values obtained as a result of applying different power supplies.

$$
\begin{aligned}
& W_{1}=\frac{Z_{\text {max }}-Z_{\text {min }}}{Z_{\text {max }}} \cdot 100 \%, \\
& W_{2}=\frac{Z_{\text {max }}-Z_{\text {min }}}{Z_{\text {mean }}} \cdot 100 \%,
\end{aligned}
$$

where: $\mathrm{bz}_{\max }-$ maximum voltage detected by the photodetector, $\mathrm{n} \mathrm{z}_{\min }$ - minimum voltage detected by the photodetector, $\mathrm{z}_{\text {mean }}$ - average voltage detected by the photodetector.

Table 1

List of calculated light flux pulsation values for all power supply methods

\begin{tabular}{lll}
\hline Power supply method & W1[\%] & W2[\%] \\
\hline Method 1 & 25.6 & 29.9 \\
\hline Method 2 & 11.7 & 11.9 \\
\hline Method 3 & 05.5 & 05.7 \\
\hline Method 4 & 98.3 & 169.6 \\
\hline
\end{tabular}

\section{Plasma temperature measurements}

The primary objective of the experiments was to examine the state of the plasma with regard to the type of ballast stabilizing the arc discharge. The study involved three ballasts controlled by three different algorithms, and one traditional electromagnetic ballast.

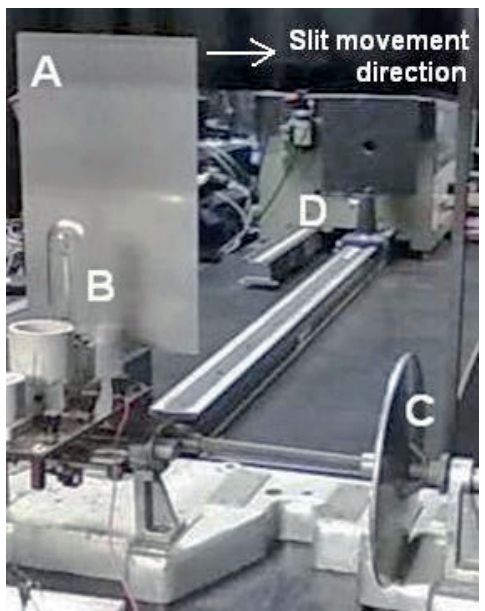

Fig. 20. View of the test stand. A - a $210 \times 350 \mathrm{~mm}$ aluminum plate with a $0,2 \times 250 \mathrm{~mm}$ slit; $\mathrm{B}-\mathrm{HID}$ lamp; $\mathrm{C}$ - the mechanism to precisely move the aluminum plate with the slit; $\mathrm{D}$ - Hilger-Engis Monospek 600 monochromator

The current waveforms of each ballast are described in Fig. 4. Experimental tests were performed with plasma diagnostics on the arc tube of a gas discharge lamp, by using optical spectroscopy. For this purpose, a special measuring system was designed. Spectral diagnostics measurement of discharge lamps leads to the determination of the radial temperature distribution along the radius $R$ of the arc tube for the different $r$ values, varying from 0 (the axis of the arc tube) to $\mathrm{R}$ (the wall of the arc tube). The direction of measurement lies along the y-direction (Fig. 3). The distance between the lamp and the aluminium plate was ca. $5 \mathrm{~cm}$. The distance between the plate and the spectroradiometer was ca. $2 \mathrm{~m}$.

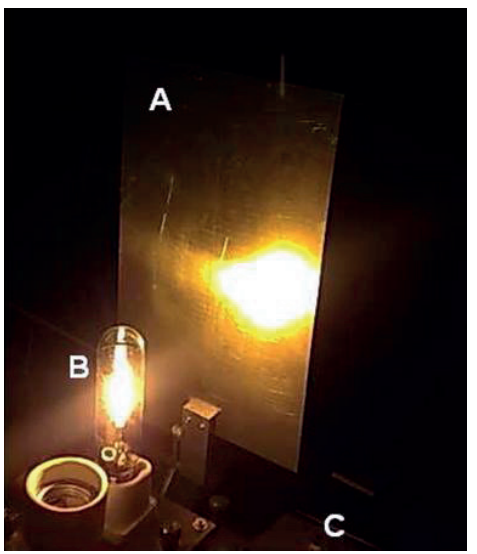

Fig. 21. View of the experiment. A - the aluminum plate with slit; $\mathrm{B}$ - HID lamp; $\mathrm{C}$ - the mechanism to precisely move the aluminum plate with the slit 
Accordingly, the device was designed to allow the reciprocal movement of the shutter along the arc tube radius (" $y$ ") in a continuous manner. As an object of the diagnostic measurement, a $70 \mathrm{~W}$ high-pressure sodium lamp was chosen. The lamp was situated in front of a movable slit. As mentioned above, the best method of plasma temperature distribution analysis in the arc tubes of high pressure sodium lamps is the examination of optically thin lines with a wavelength $\lambda=515.4 \mathrm{~nm}$ and $\lambda=616.1$ $\mathrm{nm}$, belonging to different multiplets. The results of intensity measurement along the radius of the arc tube I $v$ for the selected spectral lines from the four test ballasts, calculated on the basis of the radial distribution of the plasma temperature of the arc tube are shown in Table 1 and Table 2.

Table 2

Values of light intensity for selected wavelengths for power supply Method 1 and Method 2

\begin{tabular}{c|c|c|c|c}
\hline \multirow{2}{*}{$\begin{array}{c}\text { Distance } \\
\text { from the } \\
\text { axis of the } \\
\text { arc tube } \\
{[\mathbf{m m}]}\end{array}$} & \multicolumn{2}{|c}{$\begin{array}{c}\text { Light intensity for the selected wavelengths } \\
\text { and no. of power supply method }\end{array}$} \\
\cline { 2 - 5 } & \multicolumn{2}{|c|}{ Method $\mathbf{2}$} & \multicolumn{2}{|c}{ Method 1 } \\
\cline { 2 - 5 } & $\mathbf{6 1 6 , 1} \mathbf{n m}$ & $\mathbf{5 1 5 , 3} \mathbf{n m}$ & $\mathbf{6 1 6 , 1} \mathbf{n m}$ & $\mathbf{5 1 5 , 3} \mathbf{n m}$ \\
\cline { 2 - 5 } & $I\left[\mu W / \mathrm{cm}^{2}\right]$ & $I\left[\mu W / \mathrm{cm}^{2}\right]$ & $I\left[\mu W / \mathrm{cm}^{2}\right]$ & $I\left[\mu \mathrm{W} / \mathrm{cm}^{2}\right]$ \\
\hline-2 & 12488,83 & 2244,54 & 10822,69 & 1593,19 \\
\hline-1 & 25240,93 & 4381,76 & 20653,64 & 3154,94 \\
\hline 0 & 29550,53 & 6393,15 & 29009,58 & 4537,19 \\
\hline+1 & 13372,39 & 2605,37 & 13556,31 & 1874,46 \\
\hline+2 & 8330,69 & 1885,56 & 9142,13 & 1354,49 \\
\hline
\end{tabular}

Table 3

Values of light intensity for selected wavelengths for power supply Method 3 and Method 4

\begin{tabular}{c|c|c|c|c}
\hline \multirow{2}{*}{$\begin{array}{c}\text { Distance } \\
\text { from the } \\
\text { axis of the } \\
\text { arc tube } \\
{[\mathbf{m m}]}\end{array}$} & \multicolumn{2}{|c}{$\begin{array}{r}\text { Light intensity for the selected wavelengths } \\
\text { and no. of power supply method }\end{array}$} \\
\cline { 2 - 5 } & \multicolumn{2}{|c|}{ Method $\mathbf{4}$} & \multicolumn{2}{|c}{ Method 3 } \\
\cline { 2 - 5 } & $\mathbf{6 1 6 , 1} \mathbf{n m}$ & $\mathbf{5 1 5 , 3} \mathbf{n m}$ & $\mathbf{6 1 6 , 1} \mathbf{n m}$ & $\mathbf{5 1 5 , 3} \mathbf{n m}$ \\
\cline { 2 - 5 } & $I\left[\mu \mathrm{W} / \mathrm{cm}^{2}\right]$ & $I\left[\mu \mathrm{W} / \mathrm{cm}^{2}\right]$ & $I\left[\mu \mathrm{W} / \mathrm{cm}^{2}\right]$ & $I\left[\mu \mathrm{W} / \mathrm{cm}^{2}\right]$ \\
\hline-2 & 12239,16 & 251,66 & 6632,00 & 5765,54 \\
\hline-1 & 21509,73 & 2022,49 & 15260,81 & 11755,33 \\
\hline 0 & 35379,83 & 3458,41 & 18720,99 & 4868,51 \\
\hline+1 & 18619,83 & 6073,03 & 14709,35 & 897,02 \\
\hline+2 & 11516,68 & 2925,49 & 7197,88 & 231,49 \\
\hline
\end{tabular}

The results of the calculated radial distribution of the plasma temperature along the radius $\mathrm{R}$ of the arc tube for all power supply methods are shown in Table 4.

Table 4

Temperature of the plasma inside the arc tube for all power supply methods

\begin{tabular}{c|c|c|c|c}
\hline \multirow{2}{*}{$\begin{array}{c}\text { Distance } \\
\text { from the axis } \\
\text { of the arc } \\
\text { tube }[\mathbf{m m}]\end{array}$} & \multicolumn{4}{|c}{$\begin{array}{c}\text { Temperature of the plasma inside the arc tube } \\
\text { for all power supply methods }\end{array}$} \\
\cline { 2 - 5 } & Method 2 & Method 1 & Method 4 & Method 3 \\
\cline { 2 - 5 } & $T[K]$ & $T[K]$ & $T[K]$ & $T[K]$ \\
\hline-2 & 1352,40 & 1171,98 & 1325,36 & 718,13 \\
\hline-1 & 2733,30 & 2236,56 & 2329,27 & 1652,49 \\
\hline 0 & 3200,00 & 3141,42 & 3831,25 & 2027,17 \\
\hline+1 & 2448,08 & 2468,01 & 2016,32 & 1592,77 \\
\hline+2 & 902,12 & 989,99 & 1247,13 & 779,41 \\
\hline
\end{tabular}

The distribution of plasma temperature as a function of the radius $\mathrm{R}$ inside the arc tube for all power supply methods is presented in Fig. 22.

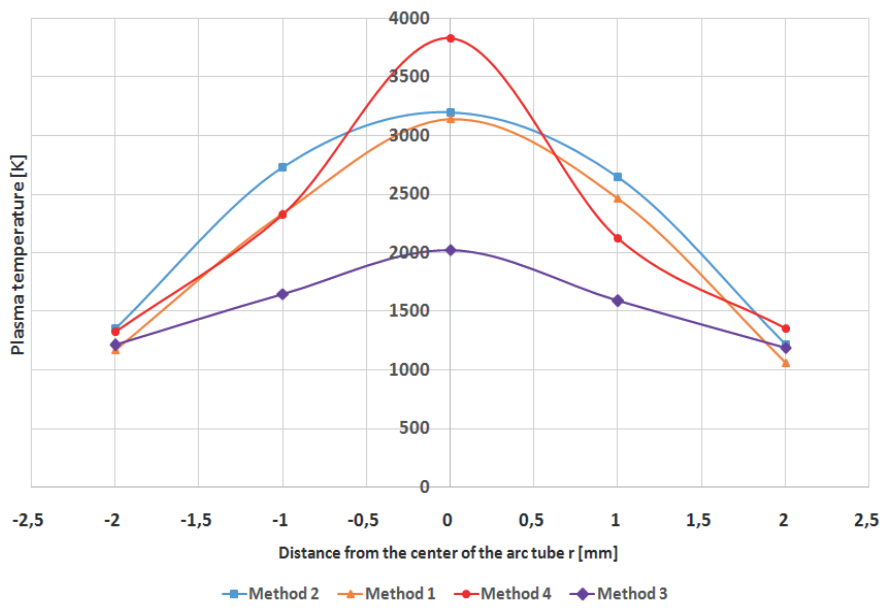

Fig. 22. Distribution of plasma temperature inside the arc tube for different power supply methods

\section{Conclusions}

As a result of the experimental work, a scheme mapping the radial temperature distribution of a $70 \mathrm{~W}$ sodium lamp, powered by three different electronic ballasts and one electromagnetic ballast has been established. Each ballast forced a different shape of current in the arc tube. The study was performed by plasma diagnostics based on emission spectroscopy, to study the changes in the intensity of radiation along the radius of the arc tube. The method used in the experiment was based on testing two optically thin lines of $\lambda=515,4 \mathrm{~nm}$ and $\lambda=616,1 \mathrm{~nm}$. The 
results of the absolute values of the radiation intensity were processed using a mathematical inversion of the Abel calculation, in order to obtain the distribution of radial temperature in the arc tube for four different test ballasts. The results show a significant influence of the choice of power supply method on the radial temperature distribution and thus for other plasma parameters, both electrical and radial. The most important results of the research are given below:

- The highest temperatures for each power supply method are in the centre of the arc tube, and vary between the methods.

- The highest temperatures of ca. $3700 \mathrm{~K}$ were obtained using Method 4. With this method, the light generated has the highest pulsation value.

- The lowest plasma temperatures of ca. $2000 \mathrm{~K}$ were obtained when powering the lamp by Method 3, that is by a high-frequency current. With this method, the light generated has the lowest pulsation.

- A significant simplification of the electronic ballast topology and control system was obtained when powering the lamp with Method 1, where the plasma temperature was ca. $3200 \mathrm{~K}$.

The results can be used as output parameters for further research on the effects of stabilization systems to optimize the performance characteristics of plasma arc discharge.

\section{REFERENCES}

[1] L. Peretto, L. Rovati, G. Salvatori, R. Tinarelli, and A.E. Emmanuel, "Investigation on the response to light flicker produced by different lamps", Proc. IEEE Instrumentation and Measurement Technology Conf., 37-42 (2006).

[2] F.J. Azcondo, A. Ortiz, M. Manana, F.J. Diaz, C. Banas, C. Renedo, S. Perez, F. Delgado, and R. Casanueva, "Effects of flicker on different types of $150 \mathrm{~W}$ high-pressure sodium lamps ballasts", Proc. IEEE Industry Applications Conf., 833-838 (2007).

[3] L. Pereto, C.E. Riva, L. Rovati, G. Salvatori, and R. Tinarelli, "Analysis of the effects of flicker on the blood flow variation in the human eye", Proc. IEEE Transactions on Instrumentations and Measurement Conf. 58 (9), 2916-2922 (2009).
[4] C.N. Moridis, A. Klados, I.A. Kokkinakis, V. Terzis, A.A. Economides, A. Karlovasitou, P.D. Bamidis, and V.E. Karabatakis, "The impact of audiovisual stimulation on alpha brain oscillations: an EEG study", Proc. IEEE Information Technology and Applications in Biomedicine Conf., 1-4 (2010).

[5] B. Lehman, A. Wilkins, S. Berman, M. Poplawski, and N. Johnson, "Proposing measures of flicker in the low frequences for lighting applications", IEEE Energy Conversion Congress and Exposition, 2865-2872, (2011).

[6] K. Chmielowiec, "Flicker effect of different types of light sources", Proc. $11^{\text {th }}$ International IEEE Conference on Electrical Power Quality and Utilisation, 1-6 (2011).

[7] J. Schwieger, M. Wolff, B. Baumann, F. Manders, and J. Sujker, "Characterization of discharge arc flicker in high-intensity discharge lamps", IEEE Industry Applications 51 (3), 2544-2547 (2014).

[8] W. Janke, A. Hapka, and M. Oleksy, "DC characteristics of the SiC Schottky diodes", Bull. Pol. Ac.: Tech. 59 (2), 183-188 (2011).

[9] S. Karyś, "Advanced control and design methods of the auxiliary resonant commutated pole inverter", Bull. Pol. Ac.: Tech. 63 (2), 489-494 (2015).

[10] T.H. Sixt, Waves in Plasmas, Springer-Verlag, New York, 1992.

[11] R. Dendy, Plasma Physics. An Introductory Course, Press Sindicate of The University of Cambridge, 1993.

[12] A. Sitenko and V. Malnev, Plasma Physics Theory, p. 432, Springer, 1994.

[13] A. Marotta, "Determination of axial thermal plasma temperatures without Abel inversion", Journal of Physics D: Applied Physics 27 (2), 268-272 (1994).

[14] M. Brambilla, Kinetic Theory of Plasma Waves. Homogeneous Plasma, p. 656, Clarendod Press, Oxford, 1998.

[15] H. Conrads, M. Schmidt, "Plasma generation and plasma sources", Plasma Sources Science Technoogy 9 (4), 441-454 (2000).

[16] T.H. Sixt, The Theory of Plasma Waves, p. 292, Literary Licensing, LLC, 2012.

[17] A.B. Mikhailovskii, Theory of Plasma Instabilites, p. 314, Springer US, New York, 2013.

[18] H. Wilhelmsson, Plasma Physics: Nonlinear Theory and Experiments, p. 513, Springer-Verlag, New York, 2013.

[19] R. Fitzpatrick, Plasma Physics. An Introduction, p. 293, Taylor Francis, United States, 2014.

[20] B. M. Smirnov, Theory of Gas Discharge Plasma, p. 423, Springer, 2015. 